\title{
Delivering elder-care environments utilizing TV-channel based mechanisms ${ }^{1}$
}

\author{
Dimitrios Amaxilatis ${ }^{a}$, Ioannis Chatzigiannakis ${ }^{\mathrm{b}, *}$, Irene Mavrommati ${ }^{\mathrm{c}}$, Evdoxia Vasileiou ${ }^{\mathrm{d}}$, and \\ Andrea Vitaletti ${ }^{\mathrm{b}}$ \\ ${ }^{a}$ University of Patras and Computer Technology Institute \& Press (CTI), Greece \\ E-mail: amaxilat@cti.gr \\ ${ }^{\mathrm{b}}$ Department of Computer, Control, and Management Engineering (DIAG), Sapienza University of Rome, Italy \\ E-mail: \{ichatz,vitaletti\}@dis.uniromal.it \\ ${ }^{\mathrm{c}}$ School of Applied Arts, Hellenic Open University, Greece \\ E-mail: \{mavrommati\}@eap.gr \\ d Spark Works ITC Ltd, UK \\ E-mail:vasiliou.evi@sparkworks.net
}

\begin{abstract}
In this paper, we present a smart environment for elderly. What makes the development of such system challenging is that the concept of smartness for elderly brings to the extreme the idea of invisibility of the technology. In our experience, elders are well-disposed to new technologies, provided that those will not require significant changes - namely, they are invisible - to their habits. Starting from this consideration, 200 caregivers responses were collected by questionnaire, so as to better understand elders' needs and habits. A system was subsequently developed allowing elders to access a number of "modern web services" as standard TV channels: at channel 43 there is the health status, at channel 45 the photos of the family, at 46 the agenda of the week, just to mention few of the available services. The content of such services is automatically generated by the smart devices in the environment and is managed by the caregivers (e.g., family members) by simple web apps. Fourteen families were asked to install the system in their house. The results of these experiments confirm that the proposed system is considered effective and user-friendly by elders.
\end{abstract}

Keywords: Internet of Things, Elder care, Ambient Assisted Living

\section{Introduction}

In recent years, we have witnessed a rapid surge in assisted living technologies due to a rapidly aging society. The aging population, the increasing cost of formal health care, the caregiver burden, and the importance that the individuals place on living independently, all motivate the development of innovative assisted living technologies for safe and independent aging. With this rapid population aging that is occurring worldwide, there is increasing interest in "smart home" technologies that can assist older adults to con-

\footnotetext{
${ }^{1}$ Footnote in title.

*Corresponding author. E-mail: ichatz@dis.uniroma1.it.
}

tinue living at home with safety and independence. Our key assumption is that a large number of elderly people are constantly supported by their children (adults) that help them with their day-to-day activities and are first to respond when bigger problems arise.

Elders have usually difficulties in adopting new technologies, and consequently, a smart environment for elders should make the technology invisible, in the sense that elders should not change their habits to take advantage of the available technology. Our approach foresees the enhancement of elders' familiar objects, such as the TV, with new and smart functionalities.

We look into cases where systems will help elders automate the activities and minimize technology jargon, thus reducing their dependence on other family 
members and caregivers. We look beyond a connected home environment where a family member or a caregiver can remotely inspect the elder's home at regular intervals. The aim is to create a smart environment that will support elders to easily carry out a number of difficult to perform activities that may require information technology skills (e.g., conduct a skype with grandkids). This is in line with the AmI research perspective reported in [6] of mediating social presence and interaction, enhancing psychological involvement and co-presence.

In this work, we present a system that facilitates awareness among families, especially for taking care of the elderly and frail living alone. Through the proposed TVSERVE platform, families and caregivers are automatically informed when the system notices pattern changes for food \& drink, medication, and other daily events. A well-defined set of innovative algorithms - details of which is outside the scope of this paper - is used in order to provide real-time intelligence and continuously analysis of the data collected to detect with high confidence any emergency situation at home. In this sense, we develop a smart assisted living environment that enables families to increase their quality time during their visits with their elders. We believe that existing hardware products are adequate for delivering an assisted living system. We are thus focusing on the software aspects of the system and be hardware-independent.

This paper is organized as follows. We start by surveying people that have a family member older than 65 years for whom they are their caregiver to understand their perspective regarding the elders' needs. In Section 2 we report our approach and main findings. Based on the input received we continue by designing a number of services that we consider important for an eldercare environment. For each service, we develop a storyboard based on which we conduct a series of interviews with a suitably selected group of caregivers. In Section 3 we summarize the feedback received on how to improve the design of elder-care services. Given these two series of interaction with end-users, we proceed by developing a prototype of an elder-care system. In Section 4 we provide a high-level description of the main components that constitute our prototype and present some key-aspects of the implementation. We conclude by assessing the usability of our prototype as perceived by the elders. In particular, in Section 5 we report the findings of our evaluation after deploying our prototype on 14 elder houses in Italy.
We believe that our attempt to develop a smart environment for elders and the approach that we followed provides valuable insights into the perception and expectations of elderly towards such a system. Indeed there is a lot of work that needs to be done in order to provide stable systems that improve the well-being of elders in their home environment. In Section 7 we conclude our work and provide some future research directions.

\section{Unserstanding caregivers' perspectives}

We start by contacting caregivers that are willing to assist us in identifying the level of technology that is more suitable for the elders that they look after. Our goal is to understand the familiarity of elders with smart home technologies. We setup a group comprised of 200 caregivers located in Italy, Germany, UK, Greece, and USA. The participants were invited to a set of interviews to identify their perspective in terms of looking after an elder. During these interviews, we informed the participants about the purpose of our study and the measures taken to ensure the participants' anonymity. We also provided a very short presentation of smart home technologies and how they can be used for setting up an ambient assisted living environment for elders.

The sample comprises of people that have an elder in their family and most of the time they are the main caregiver. Either they look after one of their parents $(53.5 \%)$ or a very close relative $(46.5 \%)$. In most cases (77.5\%) they are not the only caregiver - another family member is also supporting the elder. Their age group is mainly between $30-50$ (63.4\%), fewer are between 50-65 (22.5\%) and very few are younger than $30(14.1 \%)$. We strongly believe that the audience that participated in the survey can be considered as potential customers.

The majority of the people that participated in the survey are looking after their elder on a regular basis, and about $42 \%$ are visiting them on daily basis. On the other hand, about $22.5 \%$ are visiting the elder only a couple of days per week. Most of them are living close to them: $38.6 \%$ report that they are less than $15 \mathrm{~min}$ away, and $22.9 \%$ are about 20-30min away. From the responses received, we understand that the audience is people that interact with their elders on a regular basis and in most cases they have chosen to live close in order to have this regular interaction. 
The activities that the caregivers undertake are reported to be rather stressful. More than $52.4 \%$ find that caring for their elder family member is stressful and in some cases very stressful. Less than $15 \%$ find these activities simple to perform and easy. Interestingly, $21 \%$ strongly believe that they will consider acquiring professional caregiver services in the near future and about $43 \%$ will definitely consider it. On the other hand, about $18 \%$ do not wish to hire a professional caregiver. Based on these responses, we understand that a large part of the audience is willing to use a system to support them and in some cases completely take over the role of caregiving. Based on the responses of the interviews, we setup a questionnaire that will help us assess which categories of smart sensors are expected to be part of an assisted living environment, and which are the key features that caregivers and elders will look for. The questionnaire started with a section that lists daily activities and measured the need of the caregiver to monitoring them automatically. Then a second section included a list of smart home technologies and how important they are in setting up an assisted living environment. Finally, a third section focused on the services that the caregivers are expecting from such a system. Likert scale was used including 1: not important; 2: less important; 3: neutral; 4: important; and 5: very important.

The survey was conducted for a period of 3 days during October 2015. Almost everyone responded on the 1 st day ( $81 \%$ of participants) and very few on the 2nd and 3rd day. In the following, we summarize the responses received and evaluate the feedback.

Considering a service that will support them in their duties as caregivers, we asked them to specify the importance of a range of daily activities. In Table 1 we provide a summary of the responses received. We have ranked the responses based on the scored received, starting with those that are considered important (mark of 4) or very important (mark of 5). Remark that a neutral response gets a mark of 3, a less important a mark of 2 and a not important a mark of 1 . Based on the feedback received, we understand that the potential users are mainly interested in specific health-related aspects of the elder life. Interestingly they are positive in monitoring activities related to the diet of the elder and their weight as well as their sleeping cycles.

Regarding the technologies that the caregivers are willing to use in order to get support, almost all people involved in the survey (90\%) report that the elder's house does not have a smart home system installed, and the vast majority (78\%) are willing to install such
Table 1

Daily Activities and their importance as perceived by the target audience

\begin{tabular}{l|c|c|c|c|c||c} 
Activity & $\mathbf{1}$ & $\mathbf{2}$ & $\mathbf{3}$ & $\mathbf{4}$ & $\mathbf{5}$ & Score \\
\hline Medication & 7.4 & 8.8 & 2.9 & 26.5 & 54.5 & 81 \\
Blood pressure & 2.9 & 5.9 & 13.2 & 35.3 & 42.6 & 77.9 \\
Heart rate & 2.9 & 10.3 & 13.2 & 35.3 & 38.2 & 73.5 \\
Eating $\&$ & 7.4 & 7.4 & 17.6 & 48.5 & 19.1 & 67.6 \\
Drinking & & & & & & \\
Going out & 8.8 & 7.4 & 19.1 & 39.7 & 25 & 64.7 \\
Oven Safety & 11.8 & 11.8 & 13.2 & 33.8 & 29.4 & 63.2 \\
Sleeping & 8.8 & 11.8 & 23.5 & 36.8 & 19.1 & 55.9 \\
Toilet use & 7.4 & 17.6 & 26.5 & 38.2 & 10.3 & 48.5 \\
Activity type & 14.7 & 11.8 & 29.4 & 33.8 & 10.3 & 44.1 \\
Weight & 16.2 & 17.6 & 23.5 & 25 & 17.6 & 42.6 \\
TV watching & 19.1 & 22.1 & 30.9 & 26.5 & 1.5 & 28 \\
Walking speed & 19.1 & 23.5 & 30.9 & 17.6 & 8.8 & 26.4
\end{tabular}

a smart home system in order to improve the lifestyle of the elder that they are looking after.

Given that installing certain smart home technologies is well accepted by our target audience, we proceed by investigating which particular technology is perceived as more important. In Table 2 we summarize the technologies considered and rank them by listing those that are considered most important.

Based on the feedback received, it is evident that the potential users do not wish to install smart-home technologies in the house of the elder. The main interest is on monitoring the health for the elder and providing an emergency sensor. Apart from these technologies, other smart-home technologies are not considered important.

In the vast majority of the cases $(71.8 \%)$ the elder does not use the Internet and is not familiar with new technologies. In fact, less than $10 \%$ are frequent users of the Internet and smart technologies. We strongly believe that our assumption that the elders are not accustomed to modern online services is verified from the responses received from this survey. Developing a mobile application for a smartphone will not be easy and will alienate them from the service. It is, therefore, safe to assume that their use will be hard and consequently their adoption will be limited.

On the other hand, almost all elders $(90.2 \%)$ are frequent users of TV and more than half are watching at least 4 hours per day. Less than 3\% do not have a TV installed in their home or they do not use it at all. It is clear that TV is the device elderly use every day without problems. For this reason, we expect that providing 


\section{Table 2}

Smart Home technologies and their importance as perceived by target audience

\begin{tabular}{|c|c|c|c|c|c|c|}
\hline Technology & 1 & 2 & 3 & 4 & 5 & Score \\
\hline $\begin{array}{l}\text { Emergency } \\
\text { button }\end{array}$ & 0 & 4.4 & 25 & 25.7 & 42.6 & 68.3 \\
\hline $\begin{array}{l}\text { Automatic } \\
\text { lighting }\end{array}$ & 4.4 & 22.1 & 22.1 & 41.2 & 10.3 & 51.5 \\
\hline $\begin{array}{l}\text { Remote con- } \\
\text { trol }\end{array}$ & 8.8 & 22.1 & 27.9 & 22.1 & 19.1 & 41.2 \\
\hline $\begin{array}{l}\text { Real-time } \\
\text { monitoring }\end{array}$ & 11.8 & 14.7 & 33.8 & 30.9 & 8.8 & 39.7 \\
\hline Thermostat & 8.8 & 25 & 29.4 & 25 & 11.8 & 36.8 \\
\hline $\begin{array}{l}\text { Video moni- } \\
\text { toring }\end{array}$ & 7.4 & 10.3 & 47.1 & 26.5 & 8.8 & 35.3 \\
\hline $\begin{array}{l}\text { Activity moni- } \\
\text { toring }\end{array}$ & 11.8 & 8.8 & 44.1 & 27.9 & 7.4 & 35.3 \\
\hline $\begin{array}{l}\text { Indoor temper- } \\
\text { ature }\end{array}$ & 11.8 & 32.4 & 25 & 20.6 & 10.3 & 30.9 \\
\hline Electricity use & 14.7 & 32.4 & 27.9 & 14.7 & 10.3 & 25 \\
\hline
\end{tabular}

Table 3

Services for Elder Support and their importance as perceived by target audience

\begin{tabular}{l|c|c|c|c|c||c} 
Service & $\mathbf{1}$ & $\mathbf{2}$ & $\mathbf{3}$ & $\mathbf{4}$ & $\mathbf{5}$ & Score \\
\hline Notification & 4.4 & 8.8 & 13.2 & 33.8 & 39.7 & 73.5 \\
Routine & 1.5 & 10.3 & 16.2 & 38.2 & 33.8 & 72 \\
Change & & & & & & \\
Reminders & 1.5 & 5.9 & 22.1 & 44.1 & 26.5 & 70.6 \\
Video calls & 14.7 & 19.1 & 20.6 & 33.8 & 11.8 & 45.6 \\
Snapshot & 11.8 & 25 & 20.6 & 29.4 & 13.2 & 42.6 \\
Pictures & 35.3 & 23.5 & 17.6 & 17.6 & 5.9 & 23.5
\end{tabular}

our services as TV channels will enable easy access for the elders.

Finally, in Table 3 we try to identify which services are more attractive from the caregivers perspective. The responses received suggest that the potential users share different opinions. It seems that there is no single service that is broadly accepted as very important or not important at all.

The feedback received from this first phase of interaction with potential customers is very helpful. We have acquired valuable insights on the features that the users are expecting from an assisted living environment. We have also validated some of our key assumptions regarding our technology platform.

- Caregivers understand that providing a smartphone to the elder will not work as elders are not accustomed to this technology.
- Caregivers report that elders are definitely accustomed to the TV technology, they use it at daily basis and it would be an ideal medium for providing support.

- Caregivers are willing to acquire certain smart devices and install them at the house of the elder in order to be able to monitor some basic aspects of the daily activities of the elders.

- Caregivers do not see real value in adding too many home-automation products (e.g., smart thermostats, smart locks etc) as they do not help in providing remote caregiving.

- Caregivers understand the value of having the ability to conduct a video-conference with their elder via the TV box.

- Caregivers see added value to provide additional (related) services through the TV. However, this needs further examination in order to get more specific insights on the type of services and the exact methodology to provide them.

- Caregivers are willing to discuss the possibility of having a remote professional caregiver contacting regular the elder. However, they wish to get additional information on how this will be arranged, scheduled and how it will mix with the daily activities of the elder.

\section{Identifying key assisted living scenaria}

Given the survey conducted using the group of caregivers, we now wish to deeper our understanding of the value of an ambient assisted living environment for elders. We believe that technology should adapt to the specific needs and behavior of the elderly and not the other way round. We cannot expect that elderly will change their habits to use a technology, rather the technology should be designed to be elderly-friendly and effective at the same time.

Our point of reference is to deliver services that will support the caregivers during their daily activities via the TV available at the elder's house. Our concept is using TV channels for integrating a wider number of web services. The approach of a virtual channel model for TV is reported by [12] but referring to resources - such as broadcast, internet, storage - other than the ambient pervasive environment. Very simply, the elder can switch to specific channel numbers in order to access specific services through this very simple interface. Consider as an example at Ch1 there is the usual TV broadcast (e.g. Euronews), at $\mathrm{Ch} 2$ a Google calen- 
dar with all the relevant events, at $\mathrm{Ch} 3$ a Flickr Photo Gallery of family photos uploaded from the son etc. (see Figure 1). We expect that since elders are very familiar with the use of TV-channel paradigm, our system will also be easy use and their training will be extremely straightforward. We call this the "service as a channel" paradigm. Notice that while elderly can enjoy the simplicity of the TV-channel paradigm, relevant stakeholders (e.g., caregivers) can take full advantage of a modern web interface to access the corresponding services. Remark that in [24] a system is presented for integrating newspaper content on TV while in [26] web documents and information stored in database systems are integrated on the TV. As we will describe in the following sections, our system builds upon these approaches by integrating a wider variety of content that are focused on providing support for the caregivers.

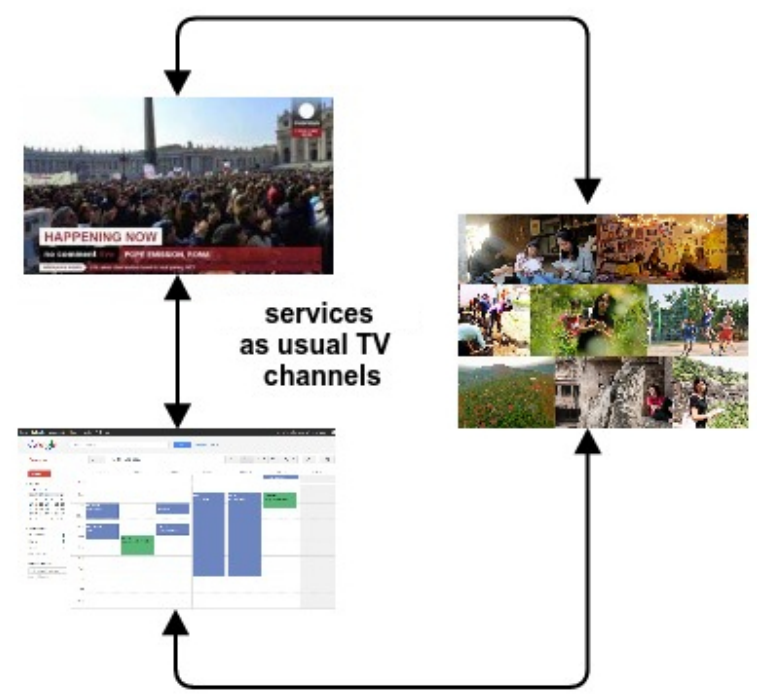

Fig. 1. Web-services accessed as TV-channels

For this reason, we design a series of services that are suitable for an elder-care pervasive health environment and that interact with the elder via the TV set. For each of these services, we design a storyboard that describes the functionality. Based on the collections of storyboard we wish to understand how professional caregivers assess their importance. For this reason we setup a focused group by carefully selecting 17 professional caregivers that work in adult daycare centers or independently. Nine (52\%) are men and eight (48\%) are women that work in Italy and Greece.

The evaluation of the storyboard was done as follows. We sent the storyboards to each caregiver and al-

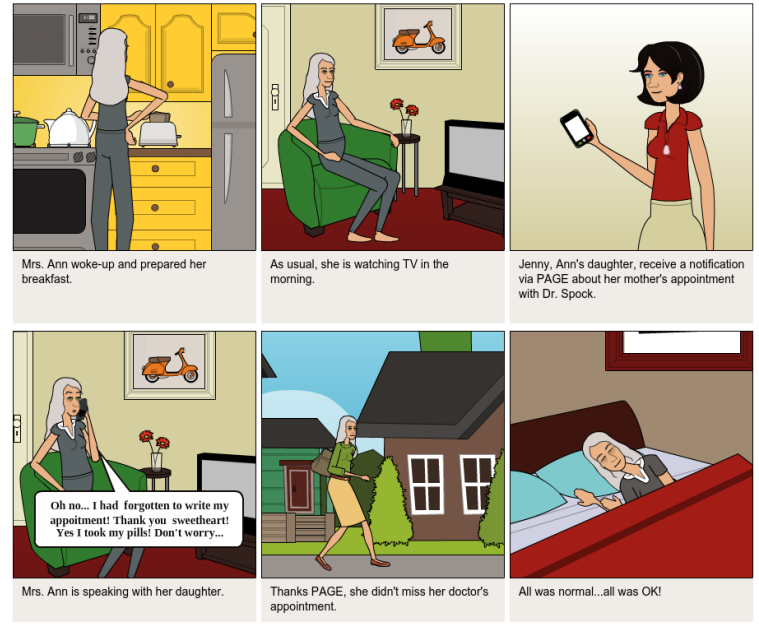

Fig. 2. Reminders \& notifications storyboard

lowed them to study them for a couple of hours. Then we conducted an individual interview during which we first asked the caregiver to describe each storyboard. During this first step, we better understood the viewpoint of each caregiver and identifying specific aspects of the services that were not properly depicted in the storyboard. We continued the interview by making certain questions regarding the value of the services, how they expect the elder to react to these scenaria and how they could be improved. We consider the above feedback very critical for the design of a successful system. In the following section, we have selected two storyboards and report the feedback received from the interviews.

\subsection{Reminders \& Notifications}

As a starting point, consider services to receive notifications about the daily medication, events scheduled for the day (social events, reminders etc), and photo albums (e.g., from their family members). In fact, we could provide a marketplace for service providers that wish to offer their services to the elder via the TV. Our primary goal is to validate if the "service-as-achannel" is adding value to the end users.

The storyboard focuses on the case of the calendar, as depicted in Figure 2. The caregiver sharing information with the elder about the medications and also meetings scheduled (e.g., doctor appointments). We use two personas: Ann, the elder living in a smart home; and Jenny, the caregiver, daughter of Ann.

Interestingly many participants responded that they already use existing services on their smartphones to 
get similar functionality. In particular, they note that since they maintain such data in the cloud, providing a view to the elder through the TV is indeed very useful. Those interviewees that do not use such web/mobile services, they mentioned that such a concept would make them seriously considering becoming users.

\subsection{Professional caregiver service}

During the survey, we assumed that smart device can be used to implement new services to assist the elders. However, we understand that certain limitations exist among smart technologies in order to provide complete monitoring of all the biometric aspects of the elder. Not only this is not easy (e.g., consider the case of monitoring whether the elder has taken the daily medications - no automated solution exists today that has achieved broad user acceptance), but it is also notpractical, in the sense that complex devices will increase the costs of the system and most importantly it might alienate the elder (e.g., due to getting a feeling that they are being watched). We, therefore, wish to look into usage scenaria where the (human) caregiver is active during the daily activities of the elder.

Instead of having smart sensors deployed all over the elder's house, we believe that providing a human contact can more easily solve the problem. In addition, the person having these regular meetings will also develop a relationship with the elder, and over time, being able to improve the quality of the services provided. In case the elder requires support or is not feeling well, the professional caregiver can dispatch the necessary support. In addition, a notification will be sent to the caregiver in order to get in touch and respond correspondingly.

In this storyboard, we wish to investigate the usefulness of integrating information exchange between the caregiver and the elder through the TV notification interface. We assume that the caregiver can communicate with the elder through the TV box by integrating messaging and video conferencing services. For example, consider the case where professional caregiving service are provided at pre-scheduled daily meetings. At fixed times during the day, the professional caregiver makes a video call to the elder to make sure that everything is normal. During these meetings, the professional caregiver is making some routine questions to the elder, in order to diagnose whether a health issue has occurred.

We encode this concept into a storyline (see Figure 3) where the elder is not feeling well. The elder

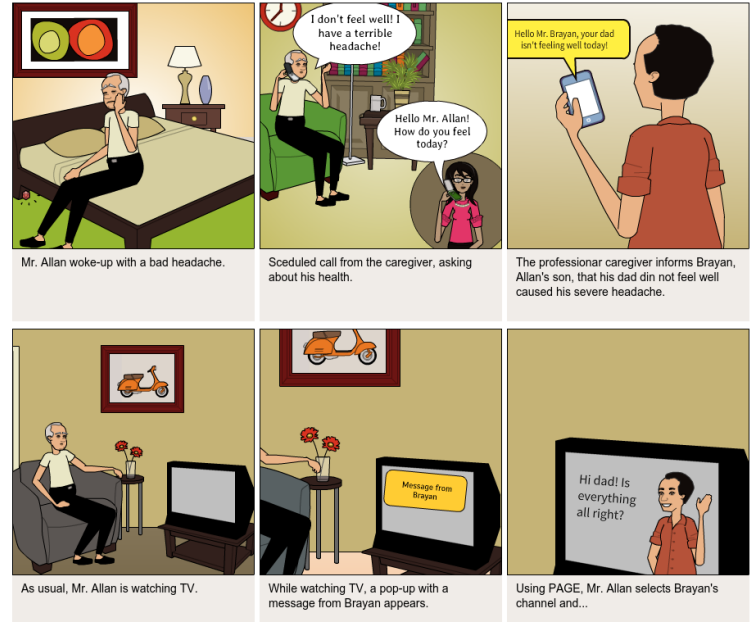

Fig. 3. Professional caregiver service storyboard

communicates the health issue during the meeting with the professional caregiver (via the TV). The professional caregiver can assess the issue, and if it requires the involvement of the caregiver, a notification is sent to his smartphone.

We received positive feedback from the majority of the participants. The interviewees found very interesting the ability to do a video-conference with their elder via the TV box. They believe that having a visual feedback will help the elder gain his confidence. Once again they find the idea of providing services through the TV channel very easy for the elder to use.

\section{High-level design \& implementation details}

After analyzing the input received from the survey and the interviews with the caregivers (both professionals and amateurs) we designed a system for delivering an ambient assisted living environment for eldercare. In our systems there are three main actors: a) the elderly, b) the caregiver, namely the person in charge of taking care of the elderly (e.g., a family member, a friend, or an institution) and c) the professional caregiver that supports the elder by providing a number of additional services.

Our system is comprised of three main elements: (a) the elder house where a number of smart devices are installed to monitor specific aspects of the elder's daily activities and a set-of-box that connects to the TV; (b) a collection of cloud-based services used for the authorization, authentication and accounting, the storage of information collected from the sensors, no- 


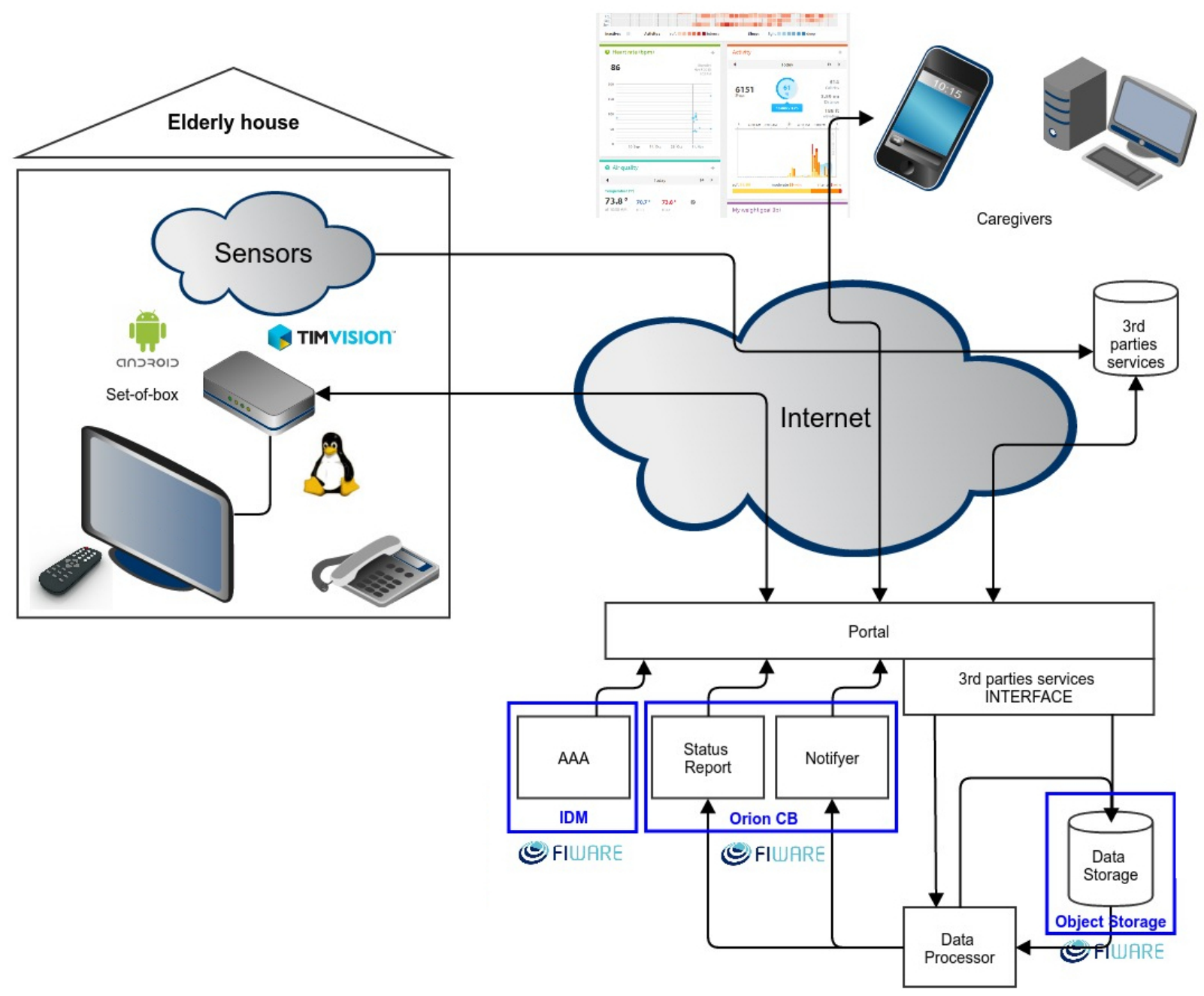

Fig. 4. Main components and their interaction

tification and complex event processing; and (c) external cloud-based services provided by 3 rd party to access and control the smart devices installed at the elder's house. Figure 4 depicts the main components of our architecture and how they interact. In Section 5 we present in details the IoT devices that we used during the evaluation of our platform, however, remark that our system is generic enough to be able to incorporate a much larger variety of devices and vendors.

According to our philosophy, the interaction with the elder is done via the TV box available at the elder's house. Therefore, all the information provided to the elder appearing as TV-channels are selected using the existing TV remote control. We do not provide any additional controller or use a camera to monitor the elder's actions. Figure 1 depicts a simple example where the elder can cycle among "conventional" TV channels, a Google Calendar and a Flickr Photo Gallery.

The smart sensors employed in our system are commercial off-the-shelve devices that provide access to the collected data via web services remotely accessible using open API. We retrieve the data using 3rd party cloud services and analyze them to identify significant events related to the daily activities of the elders. We locally store these events on our cloud-services and use them to trigger specific notification events and/or to produce periodic reports. During the evaluation of our platform (see Section 5) we used the WiThings Smart Scale $^{1}$, installed at the elder's house that transmits bio-

\footnotetext{
${ }^{1}$ http://www.withings.com/
} 


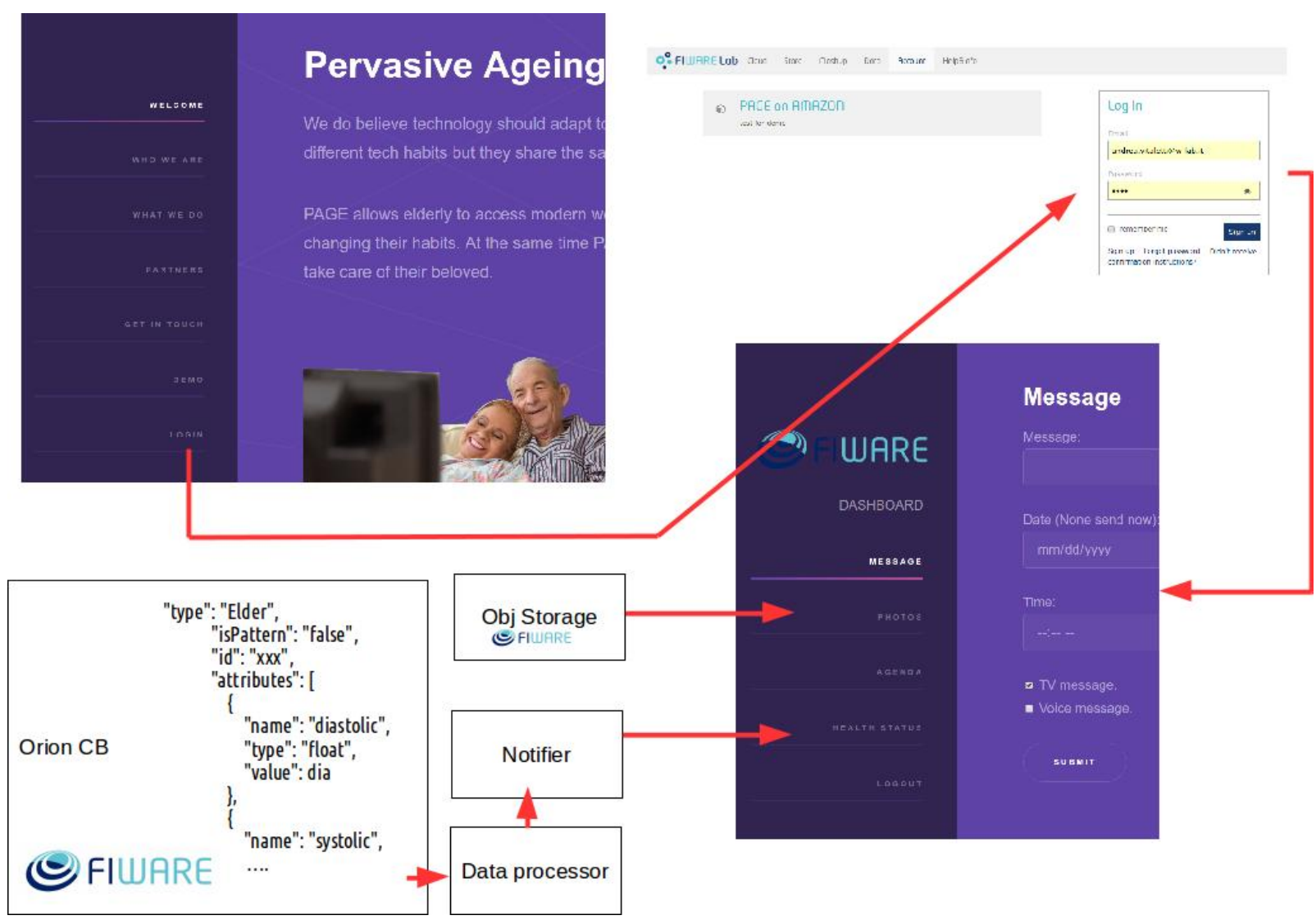

Fig. 5. Flow of information across components

metric data (e.g., weight, heart rate, and body composition measurements) to the WiThings cloud services. We retrieve these measurements from the WiThings services through the API provided ${ }^{2}$ and trigger notifications to the caregivers when we identify a specific trend on the elder's weight or when the heart rate is withing given bounds.

The caregiver (either the family member/friend or the professional caregiver) access the cloud-based services through a web portal: the central orchestrator for all the services that our platform provides. The users can access the portal at any time in order to go through the stored data utilizing their PCs or Smartphones through a unified web interface. Furthermore, the Cloud application also provides a collection of Restful APIs that enables quick integration of our platform and of its functionality with third-party systems related to (a) smart devices (e.g., commercial products by WiThings, FitBit etc.), (b) web services for deliv-

\footnotetext{
${ }^{2}$ http://oauth.withings.com/api/doc
}

ering customized content (e.g., Google Calendar and Flickr photo albums) and also (c) legacy systems, such as hospital information systems. The portal provides allows specifying notifications based on certain conditions. Through the portal, the caregiver can also communicate with the elder by sending notifications (either immediately or on specific date/time) that show up as pop-up messages on the TV of the elder. We envision that the portal will also serve for creating a broader ecosystem of caregiver services that can be selected and activated in a marketplace fashion.

Based on these basic design principles we implement a prototype of our system using the FI-WARE ecosystem of webservices [1]: a set of tools to facilitate the development of the smart application that can be configured and deployed on a cloud platform in order to easily implement an application. Currently, the FI-WARE ecosystem provides more than 50 software components (called generic technical enablers - GE) [2]. The selected FIWARE enablers provide the proper tools for implementing the envisioned architecture and functionalities with a reasonable small 
amount of effort as they provide key-role functionalities and cutting-edge technological advantages.

The FIWARE Identity Management (IdM) $)^{3}$ is used throughout our system to provide authentication to all users. Once authorized, the user accesses the portal to interact with TV box at elder's home and to get information on the status of the elder. The IdM covers a number of aspects involving users' access to services and applications, including secure and private authentication from the user's mobile devices and the web application or user profile management and privacypreserving disposition of personal data.

The Orion Context broker $(\mathrm{CB})^{4}$ is placed in the center of our platform. We use the CB to store all relevant context information taken from 3rd parties services mostly via REST interfaces. Notice that in some cases, notification services might not be available in 3rd parties services and thus a suitable polling mechanism should be implemented to feed the CB. When relevant events are identified, or the status of the elder is accessed by the caregiver, the publish/subscribe mechanism of the $\mathrm{CB}$ is used to notify the system and/or users of specific relevant events.

The Object Storage GE (OS $)^{5}$ is used to efficiently store historic records collected from the smart devices as well as relevant information, such as the photo of the family for the elder.

Finally, the interaction with the TV is done via a LINUX TV device equipped with a RealTek RTL2832U chipset. The device is capable of interacting with commercial cable-TV products, such as the TIMVISION ${ }^{6}$ set-of-box a service by TELECOM Italia with a base of users of about 500,000. In this way, elders can access services as standard TV channels using only the $\mathrm{P}+$ (next program) and P- (previous program) buttons of the remote control. Remark that the TIMVISION box is connected to the Internet via a WiFi connection.

In order to understand the interaction of the different components that constitute our system consider the following example depicted in Figure 5. The caregiver wishes to use the Portal is directed to the IdM in order to authenticate. Given that her credentials are valid, she is redirected to the protected part of the Por-

\footnotetext{
${ }^{3}$ http://catalogue.fiware.org/enablers/identity-managementkeyrock

${ }^{4}$ http://catalogue.fiware.org/enablers/publishsubscribe-contextbroker-orion-context-broker

${ }^{5} \mathrm{http}$ ://catalogue.fiware.org/enablers/object-storage-ge-fiwareimplementation

${ }^{6}$ https://www.tim.it/prodotti/tv-e-smart-living/decoder-timvision
}

tal. The Portal pulls from the OS all relevant information to the elder daily activities and presents them to the user. If the caregiver wishes to send a notification to the elder, the message is forwarded to the $\mathrm{OB}$ and through the publish/subscribe mechanism the message is forwarded to the TV box through the XMPP protocol [5]. Similarly, information retrieved from the 3rd party APIs are stored in the CB and in case a specific event is triggered, a notification is forwarded to the caregiver and/or the TV box.

\section{Usability assessment}

We now proceed by reporting the evaluation of our prototype implementation in real-world environments. We have selected a total of 14 houses located in Italy. The elders were $57 \%$ males and $43 \%$ females of age $65+$. Their age was between 65 and 80, with the majority being over the age of 72 . In each house we provided a set of off-the-shelf devices: (i) a blood pressure monitor $^{7}$, (ii) a smart body analyzer ${ }^{8}$ and (iii) a sleep monitoring environment ${ }^{9}$. These devices were properly installed and connected to the WiThings cloud system by our technical personnel.

Keeping in mind that the main challenge is to develop a system capable of providing a simplified access to modern web services to elders, in the sequel we focus on the experiences of the elders. In fact, all the involved caregivers confirmed that they have an average familiarity with modern web services and did not report any difficulties in accessing our services.

During the first meeting with the elders, we asked them to self-evaluate their confidence on using "modern technologies" such as PC and/or tablet. Interestingly, none of the participants consider him/her-self an expert user of modern technologies. About $43 \%$ of the users have absolutely no confidence in the use of modern technologies, while more than $50 \%$ have a medium or high level of confidence. All the users with very low confidence, clarify that they do not use PC or tablet at all and even if they have a smart phone, the most sophisticated activity they perform is "taking the photo that is not capable of sharing". It is worth noticing that most of the users with a medium or high level of confidence, use a very limited number of App, among

\footnotetext{
${ }^{7}$ http://www.withings.com/eu/en/products/blood-pressuremonitor

${ }^{8}$ http://www.withings.com/eu/en/products/smart-body-analyzer

${ }^{9}$ http://www.withings.com/eu/en/products/aura
} 
which the most appreciated are the meteo, photo and video sharing and whatsup, while the use of the Web for news is very limited. On the contrary, all the participants have absolutely no problem in using the TV and spend in some cases a significant number of hour per day (up to 6)in front of it. However, interestingly, all participants do not use smart applications on the TV. This observation supports our intuition on TVSERVE that the possibility of providing modern web services on the TV, using the traditional TV channel paradigm, can allow us to reach a significantly higher fraction of our target users. In the following, we report on the user experience of our participants on TVSERVE.

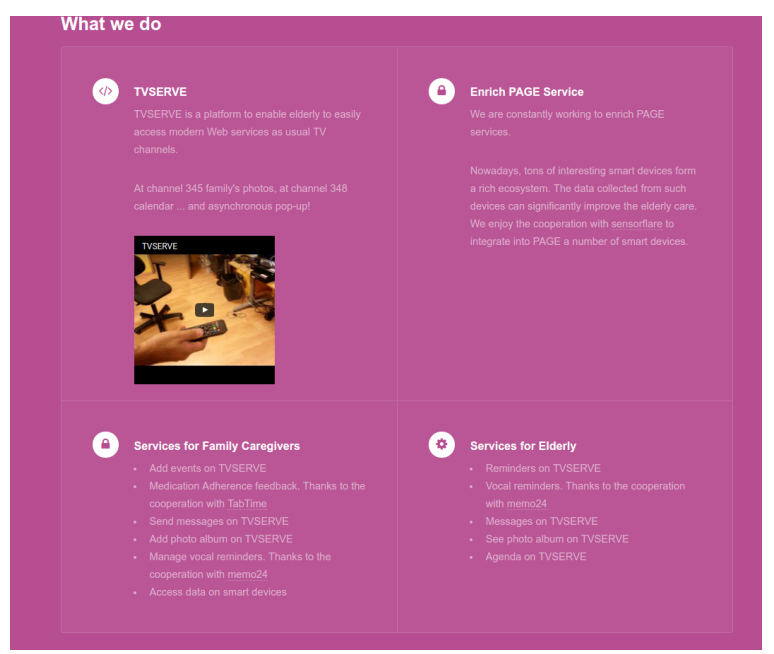

Fig. 6. The main help page as it appears as a TV channel

After this initial interview with the elders, we provided them credentials for our system (for the IdM, see Section 4) and tuned their TV to the corresponding channel showing the main help page of our system (see Figure 6). Additionally we demonstrated to the elders how to use the three smart devices provided. Then we allowed the elders to use the system for a period of 1 week. At the end of this duration, we re-visited the elders and conducted a second interview in order to evaluate the services offered in their houses.

We started by a series of questions aiming to assess the overall usability of the system. All the involved users declared that they did not experience any difficulty in accessing our services over TV. They found the approach extremely easy to use, and they stress that the possibility of using only 2 buttons (i.e. P+, P-) to access a number of services is extremely effective.

In the sequel, we continued by evaluating the services provided by the smart sensors and the TV chan-

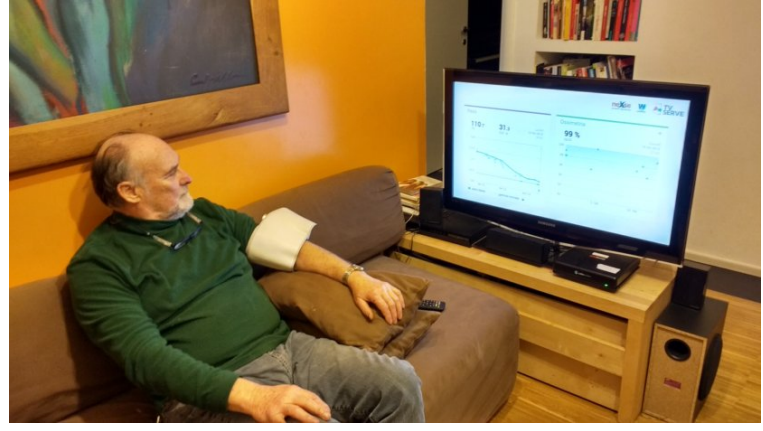

Fig. 7. A user monitors his health status on a TV channel

nels that allow elders to monitor some indicators on their health status. Figure 7 shows an example of the TV channel providing health feedback to the users. This service is highly appreciated by $40 \%$ of the users, while the remaining $60 \%$ are moderately interested. As already mentioned, our system is a collection of software services and consequently, all the necessary hardware devices have been acquired from third parties. In this particular case, all the users reported some difficulties in getting accurate measures of their blood pressure with the Whithings device. In particular, to get a reliable measure, the automatic procedure requires three readings and this is considered annoying for most of the involved users.

The next set of questions targeted the notification service. Recall that family caregivers can set-up reminders that asynchronously appears on the TV while the elder is watching TV. In our experiments, all the reminders were about medical adherence. In the example shown in Figure 8, the elder is reminded to take its daily pill foreseen for hypertension. As shown in figure 9 about $71 \%$ of the users consider this service very attractive. Interestingly, the once that are less interested in this functionality, are the ones more organized that have already a clear, regular and well-defined procedure for medicine intake. The elders pointed out that also vocal reminders over the telephone can be useful when they are not watching the TV.

Finally we evaluated the acceptance of third party web services provided to the system and in particular the Google Calendar and Flickr Photo Gallery services. For the case of the calendar service (see Figure 10), the appreciation among elders is controversial. After making additional questions, we understood that most of them have a well-established and long-term use of paper agenda. We understood that when elders already have a well-established mechanism/procedure to deal with a specific problem, the adoption of a new 


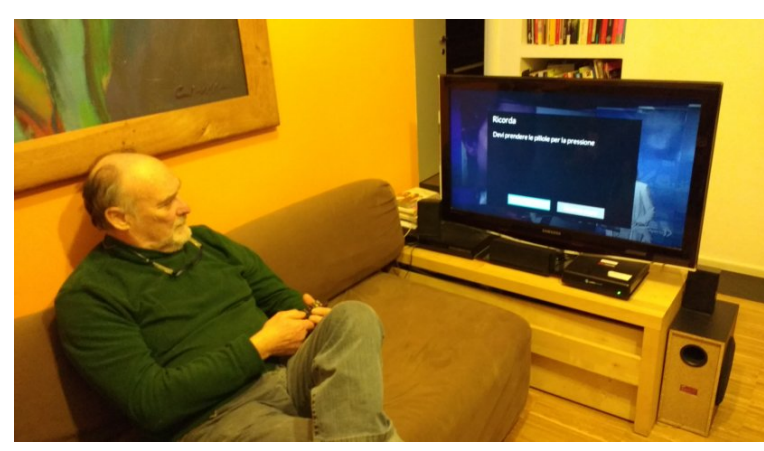

Fig. 8. A reminder appears while the user is watching the TV

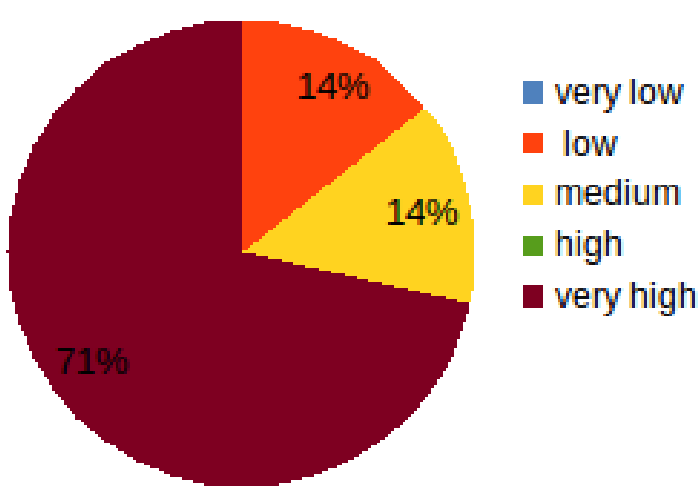

Fig. 9. Appreciation of the notification service

technological solution is difficult. For the case of the photo album service (see Figure 11), it seems that it is by far the most appreciated service. We believe that this confirms the assumption that one of the most fundamental need of the elderly is the connection with the family - a need also indicated in similar research in pervasive awareness systems [22,13]. It is worth noticing that some of the participants were particularly concerned with privacy issues: "I like very much the service, but I don't want the photos of my children are accessible online by others".

Indeed the assessment of the usability needs further experiments with a larger number of users are necessary for a deeper understanding of the acceptance of our system. However, we are confident that some useful conclusions can be drawn:

- The "service-as-a-channel" paradigm is proved to be an effective mechanism capable of simplifying the access for elderly to web services.

- All the currently available services are generally appreciated by the participants. Interestingly, more than the health-care services, the services

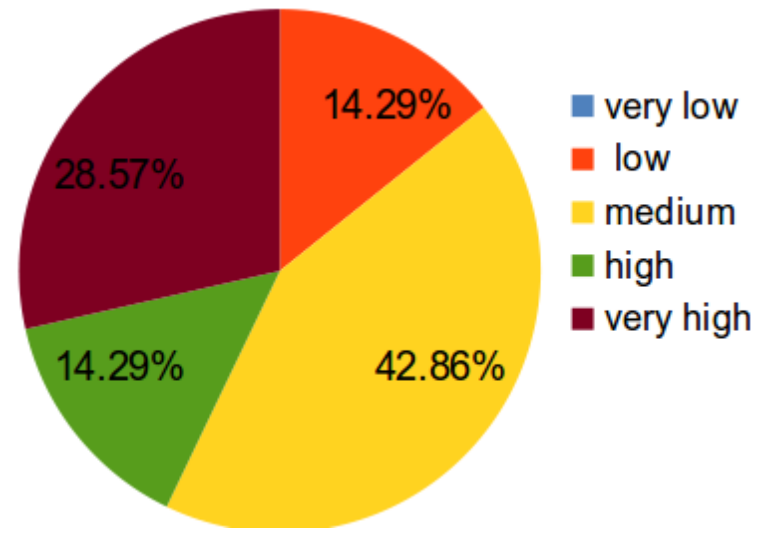

Fig. 10. Appreciation of the calendar service

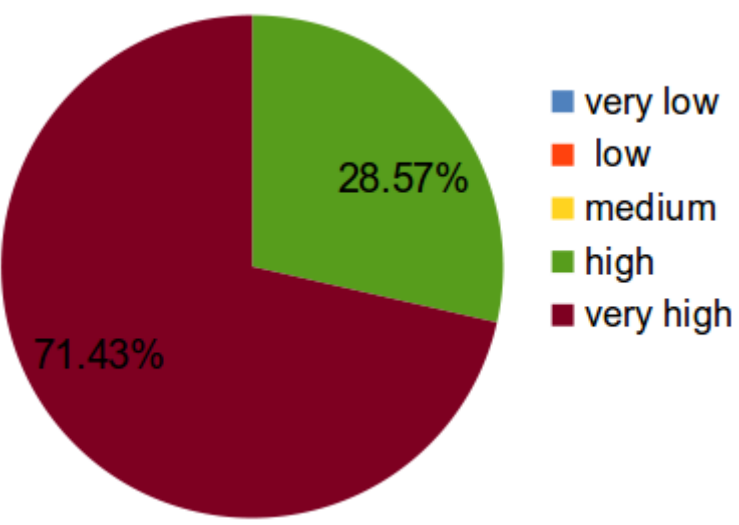

Fig. 11. Appreciation of the photo gallery service

providing some kind of connectivity with the family (e.g. photo) are the most appreciated.

- Implementing our system in commercial set-ofboxes, such as TIMVISION, is an effective strategy to reach a critical mass of users without requiring hardware or infrastructure investments except for the ones necessary for health care devices (e.g., blood pressure).

- The ease of use of health care devices is crucial for the success of such a system. Since we employ devices developed by others, we need to carefully select our hardware providers. A further investigation on the devices available in the market is necessary.

- Some users suggest the need to integrate vocal reminders in order to get reminders also when they are not watching the TV.

- When the users have strong habits (e.g., use of conventional/paper agenda), is difficult to replace 
such habits; our target users are over 65 , and change their habits is difficult.

- Our system is flexible enough to support a number of new services. The participants suggested implementing meteo and news channels.

\section{Related work}

Elder-care at home refers to pervasive systems that apply telemedicine and telehealth services in a home environment [10]. The role of health informatics in the care of elderly people and an overview of the state of the art in this field is presented in [21,18]. In [17] the authors analyze the contributions of the older users to the design of smart environments for their needs considering the findings of 21 research papers. The paper confirms that older adults are open to living in technically advanced environments if doing so would improve their quality of life and help them stay in their own homes. A classification and characterization of the main activities considered in smart home scenarios for older people and the identification of the sensors necessary to the detection of the aforementioned activities are performed in [19]. Several state-of-the-art wireless sensing systems that can be used to provide pervasive healthcare environments are presented in [8]. From all the previous work presented above we can extract significant common parameters, as the need for cost reduction in the national healthcare systems, the importance of caretakers' ease of communication with the older family members and the need for a sleek and well-established interface for elders to use and interact with despite their familiarity with technology. Additionally, it is made evident that although IoT and smart home technologies are important their diversity in interfaces and hardware specifications limits the usefulness of their usage unless an integrated system is available to use.

In [14] the authors presented a participatory evaluation involving 75 users of a smart home project implemented in an independent retirement facility. Also in that paper, the authors took a particular care to make the technology invisible: "Observational sessions confirmed that the sensors were not noticeable and residents did not change their routines.". Thus this study confirms that, also in the retirement facility, as we already observed in their own home, elders have an overall positive perceptions of the technologies provided that they do not interfere with their daily activities.
In [20] the user assessment of an assisted living platform is presented and comparatively studied with the iTriage application [3]. The study highlights some good practices regarding user interface design that is based on mobile devices. Interestingly participants were hesitant in using a tablet and would consider the alternative of using a TV set instead. Similarly, the importance of a well established and easy to use interface is noted in [25] where MedReminder, a TVbased system that reminds older users of their medication schedule, designed with the involvement of Telekom Slovenia. [16] presents Healthcare4Life a patient-centric system that combines social networking with telehealth functionalities to help monitor the well-being of elderly over the web. The overall usability of the system is something not to be ignored regardless of the age of the users as [11] highlights.

Last but not least, the Philips Motiva TV-based platform for remote patient management ${ }^{10}{ }^{11}$, has a similar approach in that, via the home-TV, it aims to provide chronic patients a familiar way to connect with healthcare professionals. Although Motiva shares resemblances with the TVSERVE approach, it aims to chronic conditions patient management, while TVSERVE is a pervasive platform that aims to facilitate family monitoring and awareness.

\section{Conclusions and Future Work}

Mark Weiser, in his paper on the origin of ubiquitous computing [27] reported at the end of the 1980s, foresaw a "physical world that is richly and invisibly interwoven with sensors, actuators, displays, and computational elements, embedded seamlessly in the everyday objects of our lives, and connected through a continuous network". Ambient Intelligence vision introduced a few years later, puts emphasis in the sensitivity and responsiveness of electronic systems to people [7], while updated AmI research perspectives are reported ten years later in [6].

Smart environments are a by-product of ubiquitous computing making human interaction with the system a pleasant experience. However, in many cases, the emphasis has been in the technical challenges of the ubiquitous computing rather than in the development of a system capable of providing a pleasant experience.

\footnotetext{
${ }^{10}$ http://www.businesswire.com/news/home/20060508005505/en/PhilipsAnnounces-U.S.-Commercial-Launch-Motiva

${ }^{11}$ http://www.hightechict.nl/projects/philips-motiva/
} 
Even technologies like Bluetooth that have been designed to be simple in terms of user perception, they are not working as they should be for numerous reasons. Although a lot of effort has been made to make Bluetooth reliable, it is still common to encounter difficulties when connecting two devices via Bluetooth for long period of time. Users that are not experienced in using the technology, find it hard to understand to overcome a difficulty, whenever it occurs, and in this sense, they are discouraged from using the technology.

Interestingly, Tablets, whether by design or simply by chance - have changed the technology landscape for seniors. Tablets, originally introduced as mobile machines (staying in contact with business, families, and friends while on the move), use multitouch technology that makes them very easy to use by elders. Arthritis and other impairments often limit a senior's fine motor skills - a disability incompatible with mouse and keyboard requirements - is now addressed by the touch screen technology, facilitating usage and minimizing user frustration. Tablets also offer an improvement over one of the most traditional form factors books. For those with poorer vision, a once - enjoyed activity like reading could be a thing of the past. Not only can those with limited mobility avoid a difficult trip to a bookstore or library by downloading a book, they can also easily adjust the text size. According to released data from Pew Research Center in a study conducted between July 18 - September 30, 2013 [23], the proportion of older adults who own either a tablet or an ebook reader is actually larger than the proportion owning a smartphone. Some $27 \%$ of seniors own a tablet, an e-book reader, or both, while $18 \%$ own a smartphone (see Figure 12).

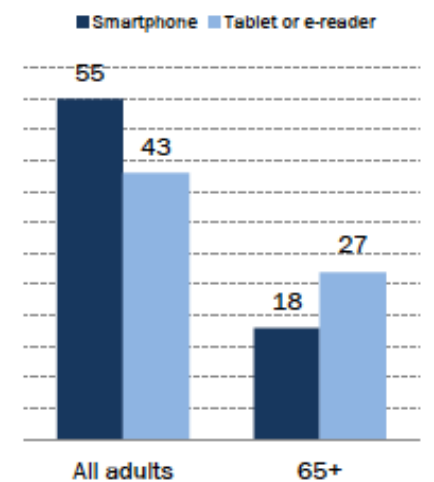

Fig. 12. Seniors are more likely to own a tablet or e-book reader rather than smartphone. Results by research conducted by Pew Research Center's Internet Project, July 18 - September 30, 2013
While Tablets are becoming a preferred technology for elders, on the other hand, Smart TVs have not attracted any attention at all. Interestingly, this is the case even though elders use their television more than any other age group [15]. According to the latest Nielsen report [4], the average level of viewing among adults who are sixty-five years of age or older is between six and seven hours per day, compared to four to five hours for younger adults. So although the television set is the dominating element of an elder's daily routine, a recent study conducted by $\mathrm{CTA}^{12}$ shows that about half of the elders indicated they do not expect to purchase a new smart TV in the foreseeable future [9]. We argue that one of the main reasons that Smart TVs have not managed to become a broadly used technology among the elders is the lack of a simplified user experience: Smart TVs are not a convenient means of connecting to the Internet and the outside world.

In this paper, inspired by recent studies that show that elderly people suffering from an illness or injury recover much more quickly at home than in a nursing facility, we investigate how to incorporate the television into a smart environment for elderly. Our starting point is that while elders are generally well disposed to explore new technologies, they are very reluctant to adopt them if they require changes to their habits. We utilize existing technologies in order to make home care easier and more reliable: devices such as the smart TV can support caregivers by assisting them in making remote care monitoring more convenient and affordable. Therefore, the main challenge in the development of such system is that the concept of smartness for elderly brings to the extreme the idea of invisibility of the technology.

Starting from this consideration, we first distributed a questionnaire caregivers to better understand elders needs and habits, and then we developed our smart system that allows elders to access a number of "modern web services" as standard TV channels. Finally, we asked 14 families to install our system in their houses and to test it in their daily life. While further experiments with a larger number of participants are likely necessary to confirm the results of this experience, however, some useful conclusions can be already drawn:

- Elders confirm that the proposed TV based system is an effective and elders-friendly solution ca-

\footnotetext{
${ }^{12}$ Consumer Technology Association
} 
pable of simplifying the access to a number of services;

- All the tested services are generally appreciated by the participants. Interestingly, more than the health-care services, the services providing some kind of connectivity with the family (e.g. access to family photos) are the most appreciated. However, when the users have strong habits (e.g. use of paper Agenda), it is difficult to replace them (i.e. an online Agenda accessible via a TV channel);

- The ease of use of the devices employed in the platform (e.g. health care devices) is crucial for their effective integration into the system.

The experience that we acquired from deploying the system into a real-world environment indicates that systems developed for elders should not expect any immediate/drastic behavioral change. Elders do not expect to change their habits and mindset so that they can use a new product, even if it was prescribed by their physician. It was apparent that their lack of willingness to make any effort to bridge the gap with new technologies must heavily influence the design of our system. In this sense we realized that services operating in a pull model fashion can be easily implemented using our $T V$-as-a-channel approach, while on the other hand, services requiring a push model approach will face significant adoption barriers. We also understood that services should keep in mind that it is hard for elders to be content providers, while on the other hand, they are extremely willing to take the role of content consumers. In this sense, the push-model is a natural fit for our approach.

Our interaction with senior adults in the context of our real-world evaluation of our system provided some interesting insights also in regards to their perception regarding privacy \& confidentiality of data. We realized that when elders accidentally become content providers they take extremely care for the privacy. Essentially, any content collected from the sensors is very sensitive to them. As a future work, we wish to look further into this observation and in particular, investigate further the levels by which the elderly are willing to participate in collective intelligence systems when compared to their interest in very customized services targeting themselves in which private information is carefully managed.

\section{Acknowledgments}

This work has been partially supported by the research project Designing Human-Agent Collectives for Sustainable Future Societies (C26A15TXCF) of Sapienza University of Rome.

\section{References}

[1] FI-WARE - an open sustainable ecosystem around public, royalty-free and implementation-driven software platform standards that will ease the development of new smart applications in multiple sectors, 2016. https://www.fiware.org/.

[2] FI-WARE catalogue. all information, documentation and tools you need as a developer to start using a generic enabler (ge) implementation, 2016. http://catalogue.fi-ware.eu/.

[3] iTriage Mobile Health, 2016. https://www.itriagehealth.com/.

[4] The Nielsen Total Audience Report: Q2 2016. Technical report, 2016. http://www.nielsen.com/us/en/insights/reports/2016/thenielsen-total-audience-report-q2-2016.html.

[5] XMPP: Extensible messaging and presence protocol, 2016. https://xmpp.org.

[6] Emile Aarts and Boris de Ruyter. New research perspectives on ambient intelligence. J. Ambient Intell. Smart Environ., 1(1):514, January 2009.

[7] Emile Aarts, Rick Harwig, and Martin Schuurmans. The invisible future. chapter Ambient Intelligence, pages 235-250. McGraw-Hill, Inc., New York, NY, USA, 2002.

[8] Hande Alemdar and Cem Ersoy. Wireless sensor networks for healthcare: A survey. Computer Networks, 54(15):2688 2710, 2010.

[9] Consumer Technology ASsociation. 16th annual CE ownership and market potential study. Technical report, 2016.

[10] Taxiarchis Botsis, George Demiris, Steinar Pedersen, and Gunnar Hartvigsen. Home telecare technologies for the elderly. Journal of Telemedicine and Telecare, 14(7):333-337, 2008.

[11] Selen Bozkurt, Neşe Zayim, Kemal Hakan Gulkesen, Mehmet Kemal Samur, Nilgun Karaağaoglu, and Osman Saka. Usability of a web-based personal nutrition management tool. Informatics for Health and Social Care, 36(4):190-205, 2011.

[12] Konstantinos Chorianopoulos and Diomidis Spinellis. User interface development for interactive television: extending a commercial $\{$ DTV $\}$ platform to the virtual channel $\{\mathrm{API}\}$. Computers \& Graphics, 28(2):157 - 166, 2004.

[13] Pavan Dadlani, Tommaso Gritti, Caifeng Shan, Boris de Ruyter, and Panos Markopoulos. SoPresent: An Awareness System for Connecting Remote Households, pages 67-79. Springer International Publishing, Cham, 2014.

[14] George Demiris, Debra Parker Oliver, Geraldine Dickey, Marjorie Skubic, and Marilyn Rantz. Findings from a participatory evaluation of a smart home application for older adults. Technol. Health Care, 16(2):111-118, 2008.

[15] Colin A. Depp, David A. Schkade, Wesley K. Thompson, and Dilip V. Jeste. Age, affective experience, and television use. American Journal of Preventive Medicine, 39(2):173 - 178, 2010.

[16] Jaspaljeet Singh Dhillon, Burkhard C Wünsche, and Christof Lutteroth. An online social-networking enabled telehealth sys- 
D. Amaxilatis, I. Chatzigiannakis, I. Mavrommati, E. Vasileiou and A. Vitaletti / Delivering elder-care environments utilizing TV mechanism 5

tem for seniors: a case study. In Proceedings of the Fourteenth Australasian User Interface Conference-Volume 139 pages 53-62. Australian Computer Society, Inc., 2013.

[17] Cynthia S. Jacelona and Allen Hansonb. Older adults' participation in the development of smart environments: An integrated review of the literature. Geriatric Nursing, 34(2):116 121,2013

[18] Sabine Koch and Maria Hägglund. Health informatics and the delivery of care to older people. Maturitas, 63(3):195 - 199, 2009.

[19] Qin Ni, Ana Belen Garcia Hernando, and Ivan Pau de la Cruz. The elderly's independent living in smart homes: A characterization of activities and sensing infrastructure survey to facilitate services development. Sensors, 15(5):11312, 2015

[20] Christos Panagopoulos, Eirini Kalatha, Panayiotis Tsanakas, and Ilias Maglogiannis. Evaluation of a mobile home care platform - lessons learned and practical guidelines. In Ambient Intelligence - 12th European Conference, AmI 2015, Athens, Greece, November 11-13, 2015, Proceedings, volume 9425 of Lecture Notes in Computer Science, pages 328-343. Springer, 2015.

[21] Parisa Rashidi and Alex Mihailidis. A survey on ambientassisted living tools for older adults. Biomedical and Health Informatics, IEEE Journal of, 17(3):579-590, 2013.

[22] Natalia Romero, Joy van Baren, Panos Markopoulos, Boris de Ruyter, and Wijnand IJsselsteijn. Addressing Interpersonal Communication Needs through Ubiquitous Connectivity:
Home and Away, pages 419-429. Springer Berlin Heidelberg, Berlin, Heidelberg, 2003.

[23] Aaron Smith. Older adults and technology use. Technical report, 2014. PewResearch Center. http://www.pewinternet.org/2014/04/03/older-adults-andtechnology-use/.

[24] Caj Södergård, Matti Aaltonen, Sari Hagman, Mikko Hiirsalmi, Timo Järvinen, Eija Kaasinen, Timo Kinnunen, Juha Kolari, Jouko Kunnas, and Antti Tammela. Integrated multimedia publishing: combining $\{\mathrm{TV}\}$ and newspaper content on personal channels. Computer Networks, 31(11âĂŞ16):1111 1128, 1999.

[25] Emilija Stojmenova, Bojan Imperl, Tomaž Žohar, and Dejan Dinevski. Adapted user-centered design: a strategy for the higher user acceptance of innovative e-health services. Future internet, 4(3):776-787, 2012.

[26] Katsumi Tanaka, Akiyo Nadamoto, Machiko Kusahara, Taeko Hattori, Hiroyuki Kondo, and Kazutoshi Sumiya. Back to the $\mathrm{TV}$ : information visualization interfaces based on tv-program metaphors. In 2000 IEEE International Conference on Multimedia and Expo, ICME 2000, New York, NY, USA, July 30 - August 2, 2000, pages 1229-1232. IEEE Computer Society, 2000.

[27] Mark Weiser, Rich Gold, and J.S. Brown. The origins of ubiquitous computing research at parc in the late 1980s. IBM Systems Journal, 38(4):693-696, 1999. 